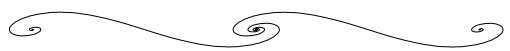

\title{
Experimental Techniques for Investigating Chaos in Electronics
}

\author{
Chi K. Tse ${ }^{1}$ \\ Department of Electronic and Information Engineering \\ The Hong Kong Polytechnic University \\ Hong Kong, China \\ encktse@polyu.edu.hk
}

\begin{abstract}
In the study of nonlinear phenomena in electronics, experiments are indispensable for the purpose of verifying the analytical findings and simulation results. This chapter begins with a discussion on the roles of experimental measurements and computer simulations in the study of nonlinear systems. A tutorial overview of the commonly used laboratory techniques for studying nonlinear phenomena in electronic circuits is given. Specifically, some techniques for displaying phase portraits, Poincaré sections and bifurcation diagrams on the oscilloscope are discussed.
\end{abstract}

\footnotetext{
${ }^{1}$ This work is supported in part by the Hong Kong Research Grant Council under a competitive earmarked grant (No. PolyU-5131/99E).
} 


\subsection{Introduction}

Sensitive dependence on initial conditions and lack of long-term predictability are key features of chaotic systems, which have profound implications on the approaches taken to study such systems. From the computational standpoint, exact trajectories cannot be sought for a chaotic system, no matter how accurate the numerical simulations and the models used in the simulations are. Any computed trajectory will "eventually be wrong". This is particularly true with modern digital computers which introduce round-off errors, and depending upon the algorithms used, the errors can accumulate and render any solution eventually inaccurate. This raises some doubts as to how much we can trust our model (for a particular system). In particular, we may ask several questions: How well does the model describe reality? And under what conditions? Is it a physical model reflecting the processes in the systems or a phenomenological model reproducing phenomena, waveforms, etc. We may use an analytical approach and try to identify stability regions, equilibria, conditions for bifurcations, etc. Alternatively we may perform numerical studies giving only quantitative results.

An equally important mode of investigation of nonlinear phenomena in electronic circuits and systems is to begin with experimentation. Certain phenomena may be observed unintentionally while developing a practical electronic system. The quest for an explanation for the observed unusual behavior motivates in-depth analysis of the underlying mechanism. This finally calls for appropriate analytical models which fit the observed phenomena and provide adequate analytical basis to predict the occurrence of similar phenomena.

\subsection{Overview of Simulation Study and Verification}

For the purpose of studying chaotic systems, very often, analytical models need not be very accurate since exact trajectories are never wanted. What is needed is perhaps a simple model that contains adequately the salient nonlinear features of the system under study. After all, sensitive dependence will strike in and render the model "useless for generating exact trajectories". Fortunately, what we want the model to predict is really qualitative behavior such as bifurcations and exhibition of chaotic attractors. If simulations are performed to verify the predicted qualitative behavior or to study certain behavior, they must be viable ones in order to reflect the true behavior of the system. Thus, using the same mathematical model to simulate the system can only be regarded as part of the analysis (which is done numerically) and should not be claimed as a verification or simulation study. For electronic circuits, any viable verification or computer simulation study, should be performed using real circuit models. 
Some existing packages such as PSPICE may help in this respect.

Computer simulation alone, however, is not completely convincing as a verification or investigation tool since numerical procedures are always subject to round-off errors, however small, and the model used for simulation may not fully describe the system. What we see in the computer simulated waveforms may sometimes contain artifacts due to numerical errors or flaws in the simulating model. Hence, laboratory experiments remain an indispensable form of verification. Furthermore, as mentioned earlier, experimentation can sometimes be well ahead of any analysis and simulation, particularly for many practical electronics circuits whose popularity in practical use often precedes any detailed analysis.

In summary, rigorous analysis, viable simulations and laboratory experiments are all indispensable, and they complement one another $[1,2]$. In this chapter we focus our attention on experimental investigation, and specifically on some essential laboratory techniques for capturing Poincaré sections and bifurcation diagrams. It should be mentioned that the literature abounds with various techniques for capturing nonlinear behavior in electronics [4]- [8] and the ones shown in this chapter represent only a few possibilities.

\subsection{Experimental Investigation}

From what has been said, experimental study plays the dual role of verifying and establishing certain nonlinear phenomena in physical systems. It thus becomes obvious that experiments should be designed to focus the kinds of investigation that would be used in analysis and/or simulations. Usually, we examine nonlinear phenomena in one or more of the following aspects:

1. Time-domain waveforms

2. Phase portraits

3. Frequency spectra

4. Poincaré sections

5. Bifurcation diagrams.

While time-domain waveforms, phase portraits and frequency spectra are familiar to most electronic engineers, the way to obtain Poincaré sections and bifurcation diagrams on the oscilloscope may appear non-trivial. Nonetheless, we will briefly review in the next section the commonly used instruments for capturing time-domain waveforms, phase portraits and frequency spectra, and then will go into details of the various laboratory techniques for displaying Poincaré sections and bifurcation diagrams on the oscilloscope. It should be noted that the methods described here represent only a few possibilities that 


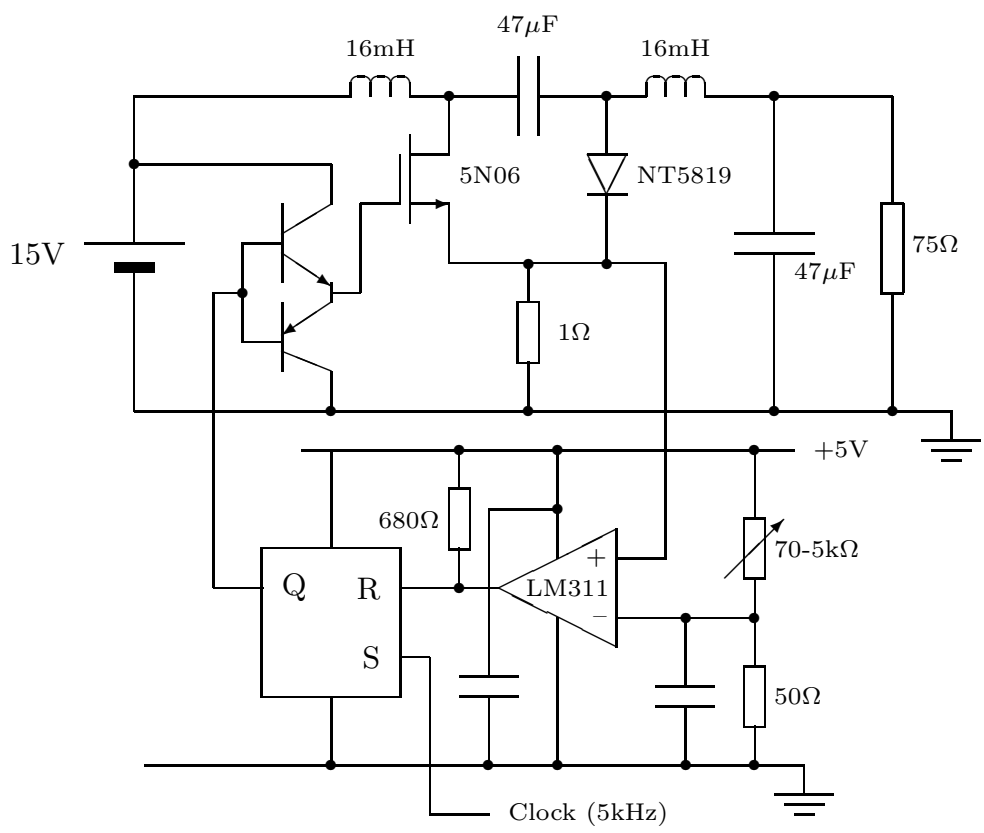

FIGURE 18.1

An experimental dc/dc converter (Ćuk type) under fixed-frequency current-mode control. The RS flip-flop block is constructed from a pair of NOR gates [9].

have proven to work, but they are not necessarily the best methods for capturing nonlinear behavior of electronic circuits.

\subsection{Displaying Time-domain Waveforms, Attractors and Spectra}

It should be straightforward enough for most engineers to capture periodic waveforms using an analog oscilloscope. For aperiodic waveforms such as those of quasi-periodicity or chaos, the waveforms appear to be shaking — which is generally a signature of these nonlinear phenomena. If a digital storage oscilloscope (DSO) is used, one can 'freeze' the waveform at a certain instant and then the irregular behavior of a chaotic waveform becomes apparent. To display phase portraits, one can simply use the $\mathrm{X}-\mathrm{Y}$ mode of the oscilloscope instead of a sweeping time base [3].

The phase portraits provide a handy tool to identify chaotic behavior. Chaos is characterized by phase portraits that cover a well defined bounded region, and 


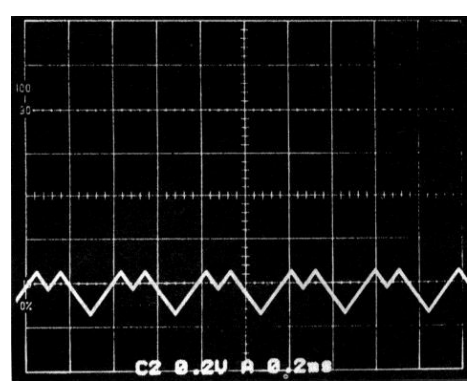

(a)

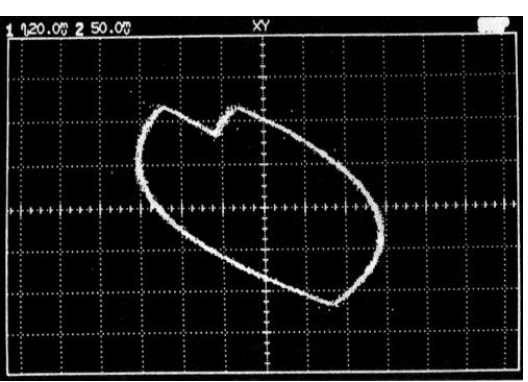

(b)

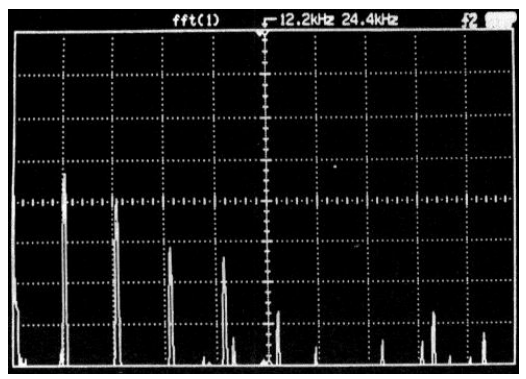

(c)

FIGURE 18.2

Experimental waveform, phase portrait and frequency spectrum from oscilloscope for a Ćuk dc/dc converter operating under current-mode control showing period-2 operation. Reference for $i_{1}+i_{2}$ set at 0.49A. (a) Inductor current $(1 \times 0.2 \mathrm{~A} / \mathrm{div}, 0.2 \mathrm{~ms} / \mathrm{div}$, lowest horizontal grid line is $0 \mathrm{~A})$; (b) phase portrait of inductor current against a capacitor voltage; (c) FFT of inductor current.

can be easily distinguished from random noise which shows fuzzy edges on phase portraits. Furthermore, chaotic signals are wide-band signals, and hence can be easily distinguished from periodic signals by inspecting their frequency spectra. This can be done with a spectrum analyzer. Moreover, some DSO's actually provide spectral analysis by performing the so-called Fast Fourier Transform (FFT) on the signal being measured. Thus, we may use a DSO to obtain frequency spectra for periodic and non-periodic waveforms.

As an example, we consider a Ćuk dc/dc converter operating under fixedfrequency current-mode control [9]. Figure 18.1 shows the experimental converter circuit which can be constructed and tested in the laboratory without much difficulty. The operation of the circuit can be briefly described as follows. The essential control variable is the sum of the two inductor currents which is 


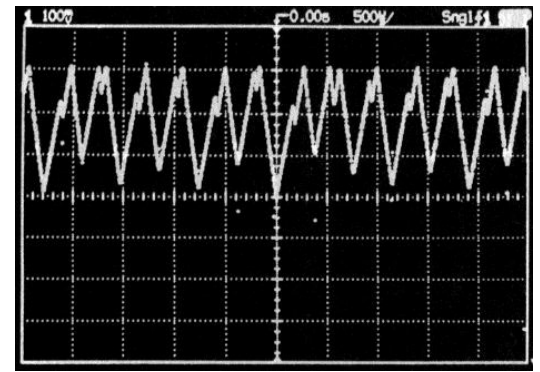

(a)

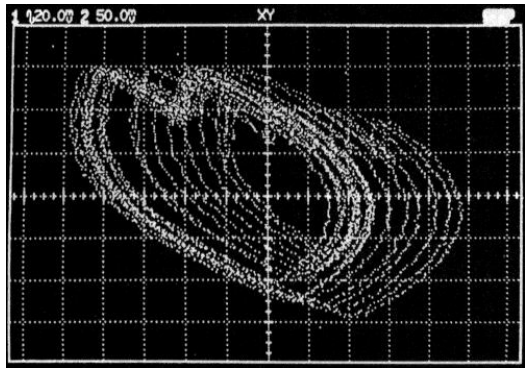

(b)

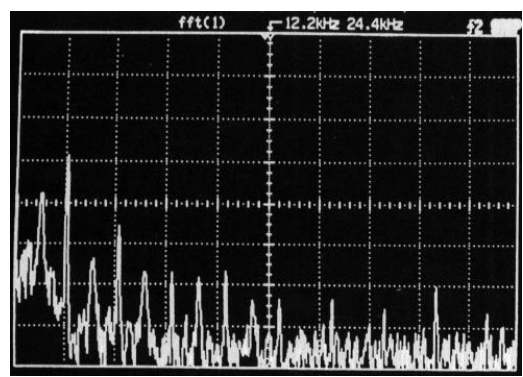

(c)

FIGURE 18.3

Experimental waveform, phase portrait and frequency spectrum from oscilloscope for a Ćuk dc/dc converter operating under current-mode control showing chaotic operation. Reference for $i_{1}+i_{2}$ set at $0.74 \mathrm{~A}$. (a) Inductor current $(1 \times 1 \mathrm{~V} / \mathrm{div}, 500 \mu \mathrm{s} /$ div, lowest horizontal grid line is $0 \mathrm{~A})$; (b) phase portrait of inductor current against a capacitor voltage; (c) FFT of inductor current.

picked up by the $1 \Omega$ sensing resistor. The voltage across this sensing resistor is then compared with an adjustable threshold voltage which serves as a bifurcation parameter. The on-off status of the power switch (5N06) is determined by the output of the comparator (LM311). Essentially, when the power switch is on, the voltage across the sensing resistor ramps up, and as it reaches the threshold voltage, the RS flip-flop (actually a pair of NOR gates) is re-set and the power switch is turned off. Then, the control variable ramps down, until the clock pulse sets the RS flip-flop again and turns the switch back on. The cycle repeats at $5 \mathrm{kHz}$. Analysis has shown that changing the parameter values affect the qualitative behavior of the system. Here, we include in Figs. 18.2 and 18.3 some typical waveforms, phase portraits and frequency spectra obtained from this circuit. 


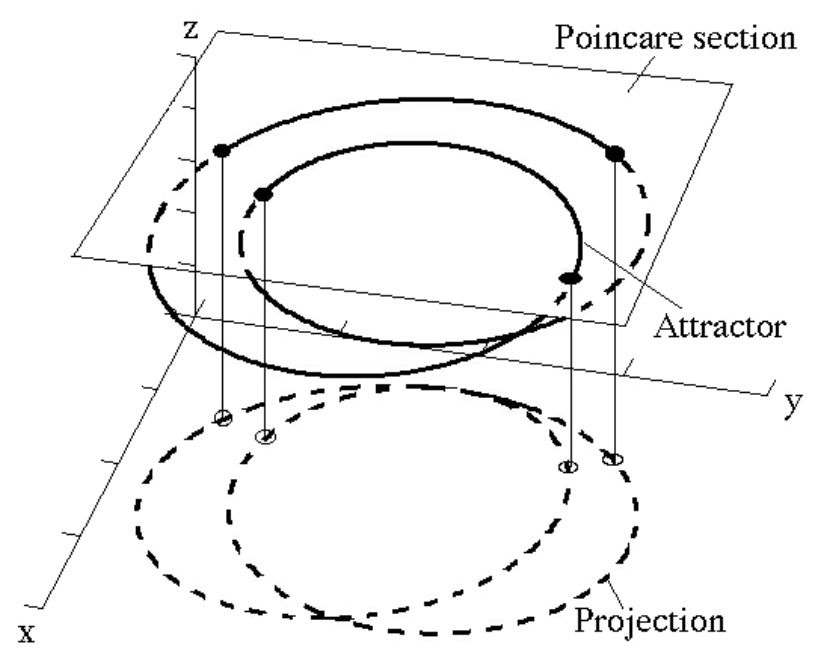

FIGURE 18.4

An attractor and Poincaré section.

\subsection{Displaying Poincaré Sections}

One approach to studying nonlinear systems is to examine a Poincaré section of a trajectory. To keep our discussion simple, we consider initially thirdorder autonomous circuits. For simplicity we define a Poincaré section as a 2-dimensional (2-D) plane that intersects the trajectory.

By examining the way the steady-state trajectory (sometimes referred to as the attractor) intersects the Poincaré section, one can tell if the steady-state motion is periodic, quasi-periodic or chaotic $[10,11]$. The following is what we will typically see on a Poincaré section. First, if the motion is periodic, we will see a finite number of points on the Poincaré section. If the motion is quasi-periodic (torus), we will see a closed loop on the Poincaré section. And if the motion is chaotic, we will see a large number of irregularly and densely located points on the Poincaré section.

\subsubsection{Principle of Poincaré section measurement}

Obviously, since the oscilloscope can only display 2-D phase portraits, we can at best view a projection of an attractor. Using the X-Y mode of the oscilloscope, we can display a 2-D projection (effectively a phase portrait) from any two given signals. This is adequate as long as the 2-D projection clearly reflects the kind of the attractor. For most cases, we are still able to tell, from a 2-D 


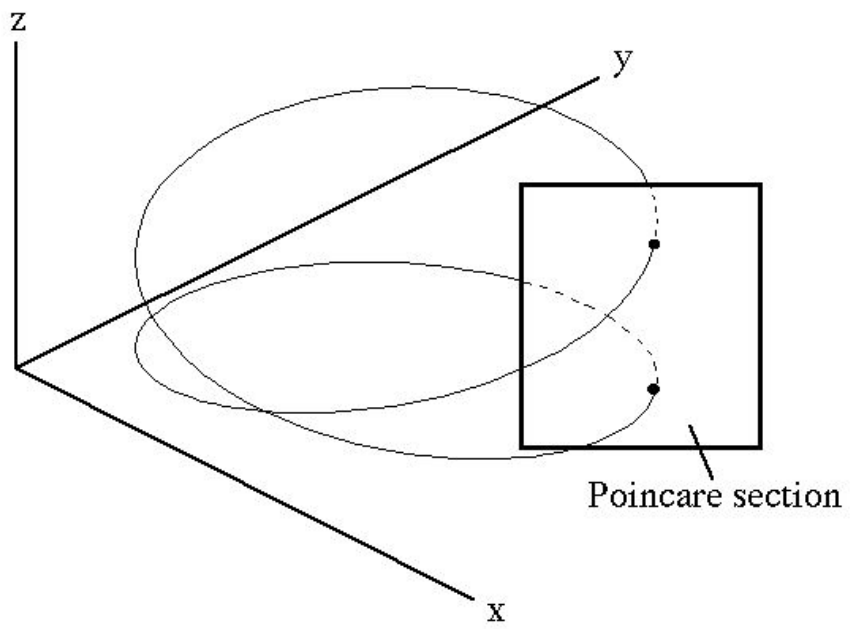

(a)

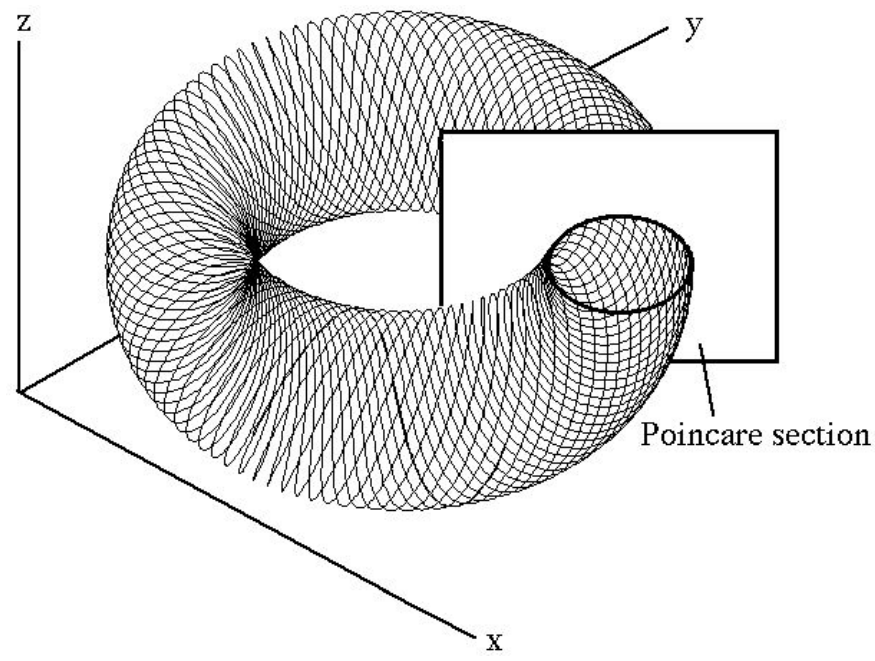

(b)

FIGURE 18.5

Poincaré section of (a) a period-2 orbit; and (b) a quasi-periodic orbit. 


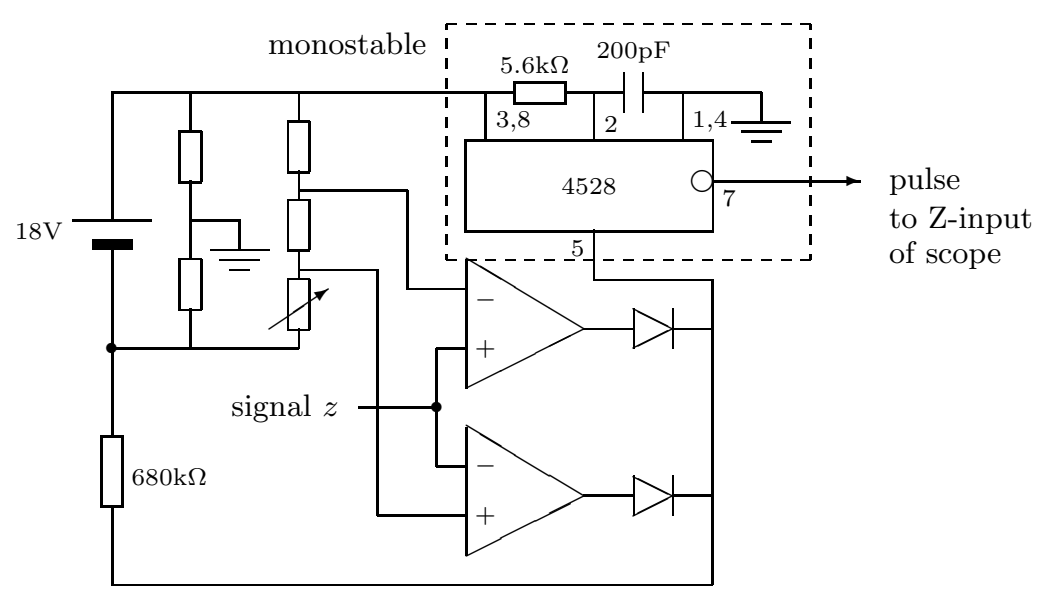

FIGURE 18.6

Circuit for detecting intersection of attractor and Poincaré section.

projection, confidently if it is a periodic orbit. However, for a torus or chaotic attractor, we usually cannot make a definite conclusion unless we know how its Poincaré section looks like. Fortunately it is not difficult to show a Poincaré section on the oscilloscope along with the 2-D projection of the attractor. What we need to do is to highlight the attractor when it cuts through a certain 2-D plane which has been chosen as the Poincaré section.

Suppose the system's variables are $x, y$ and $z$, and the oscilloscope is now plotting $x$ against $y$ using the $\mathrm{X}-\mathrm{Y}$ mode [3]. Thus, the oscilloscope is showing the projection of the attractor on the $\mathrm{x}-\mathrm{y}$ plane. We may define a Poincaré section as $z=k$, where $k$ is a suitable constant. Imagine that the attractor is traversing in the 3-D space and is cutting through the plane $z=k$ in both upward and downward directions, as shown in Fig. 18.4. Further suppose that we have a means to highlight the intersecting points on the projection. (We will explain how to do it later.) If the motion is periodic, such as the one shown in the figure, the projection should adequately reflect the periodicity of the motion. In this case, we see stationary points on the projection being highlighted.

It should be noted that, by definition, the Poincaré section captures only one direction of crossing so that the period, if finite, can be correctly found. In a period-2 orbit as shown in Fig. 18.5 (a), for example, the Poincaré section should correctly show only two crossing points, instead of four. Moreover, if the motion is quasi-periodic, we should see a closed loop on the projection, as shown in Fig. 18.5 (b), and likewise for chaotic motion. 


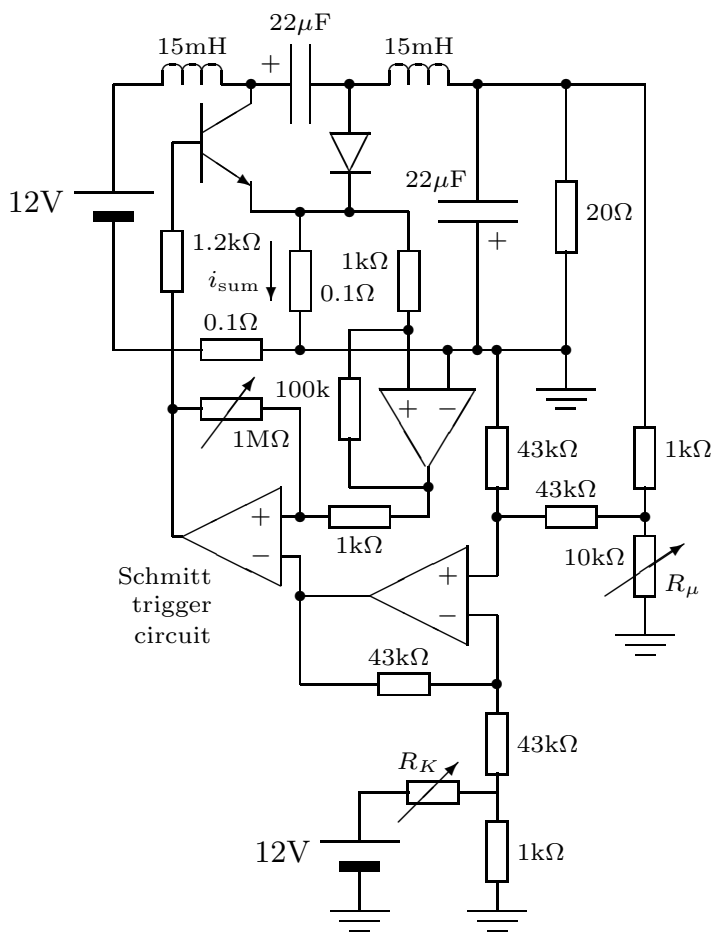

FIGURE 18.7

Experimental circuit of free-running autonomous Ćuk converter.

Clearly, we need a comparator circuit to determine when the attractor is hitting the plane $z=k$. This can be done easily using the circuit shown in Fig. 18.6. The function of this circuit is to produce a pulse whenever the signal $z$ is equal to the value $k$ which is set by a potentiometer. The display of the Poincaré section is then left to the oscilloscope. The idea is to make use of the Z-axis modulation function of the oscilloscope, which momentarily brightens the trace when its $\mathrm{Z}$-input receives a pulse. Thus, if the output from the circuit described above is applied to the Z-input of the oscilloscope, the trace will momentarily brighten whenever the attractor intersects the plane $z=k$. This technique was also used by Deane and Hamill [12] in their experimental study of chaos in power electronics circuits. 


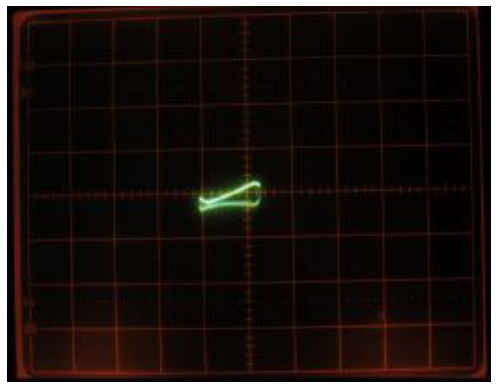

(a)

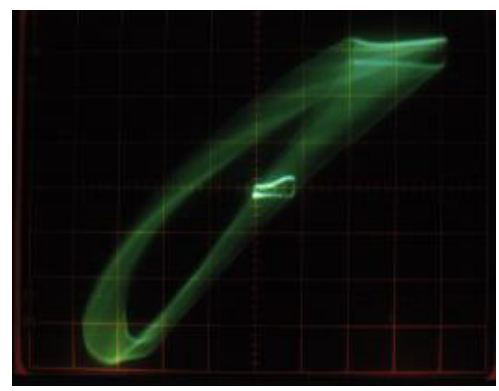

(c)

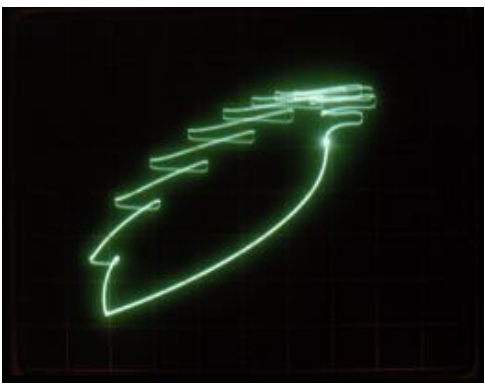

(b)

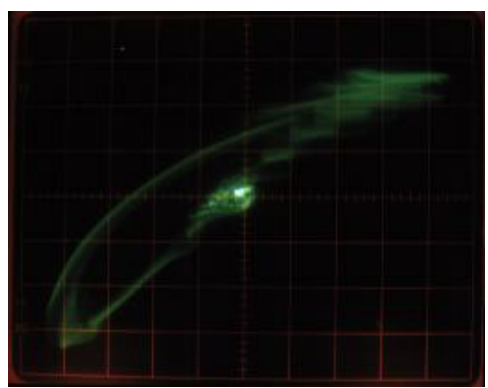

(d)

FIGURE 18.8

Phase portraits from autonomous Ćuk converter showing (a) fixed point; (b) limit cycle; (c) quasi-periodic orbit; (d) chaotic orbit. Poincaré section highlighted in (b), (c) and (d). The output voltage across the $20 \Omega$ load is used as input to the Poincaré section detector circuit of Fig. 18.6.

\subsubsection{Example: An autonomous dc/dc converter}

As an example, we consider a third-order autonomous Ćuk dc/dc converter [13]. The experimental circuit is shown in Fig. 18.7. This circuit operates under a so-called free-running current-mode control which is effectively a bang-bang type of control. The sum of the inductor currents, sampled by a $0.1 \Omega$ sensing resistor, is compared with a reference signal which is derived continuously from the output voltage via a feedback circuit. The comparison is actually done by a Schmidt trigger circuit which also provides adjustment for the width of the hysteretic band. Referring to the circuit diagram of Fig. 18.7, the feedback voltage gain is adjusted by $R_{\mu}$ and the inductor dc current level is adjusted by $R_{K}$. The $1 \mathrm{M} \Omega$ variable resistor sets the width of the hysteretic band and hence the switching frequency. 
The analysis of the dynamics of this converter reveals the possibility of a Hopf bifurcation, and computer simulation consistently reveals the characteristic sequence of changes in qualitative behavior starting from fixed point, via limit cycles and quasi-periodic orbits, to chaos [13]. Experimental study would inevitably require examining Poincaré sections since quasi-periodic and chaotic attractors can only be distinguished from the appearance of their Poincaré sections. Figure 18.8 shows the sequence of phase portraits starting from fixed point, through limit cycle and quasi-periodic orbit, to chaotic orbit.

\subsubsection{Poincaré sections for non-autonomous circuits}

For non-autonomous systems, Poincaré sections can be obtained in a likewise manner with the Z-axis modulation set to sample at the switching frequency of the converter under study. The resulting display contains bright dots along with the attractor, and the number of bright dots indicates the period of repetition in the case of periodic and subharmonic motion. Specifically, $N$ bright dots means that the system is attracted to a subharmonic orbit whose period is $N$ times the switching period. A large number of irregularly and densely located points may indicate chaos.

\subsection{Displaying Bifurcation Diagrams}

Bifurcation diagrams are frequently used for identifying the way in which a system's qualitative behavior changes as some chosen parameters are varied. To display a bifurcation diagram, we need to construct a circuit which generates the necessary signals to the oscilloscope for displaying a bifurcation diagram. We will begin with basic operational requirements and then discuss the details of the implementation. For brevity, we will refer to the electronic circuit being studied as system under test (SUT).

\subsubsection{Basic operational requirements}

We first examine what a bifurcation diagram contains. A typical bifurcation diagram, as shown in various sections of this book, has its horizontal axis corresponding to variation of a bifurcation parameter and its vertical axis corresponding to the sampled steady-state value of a variable from the SUT. Obviously, we can make use of the $\mathrm{X}-\mathrm{Y}$ mode of the oscilloscope to display a bifurcation parameter provided the necessary signals are applied to the $\mathrm{X}$ and $\mathrm{Y}$ input channels. In order to generate these signals, we need to perform two basic processes: 


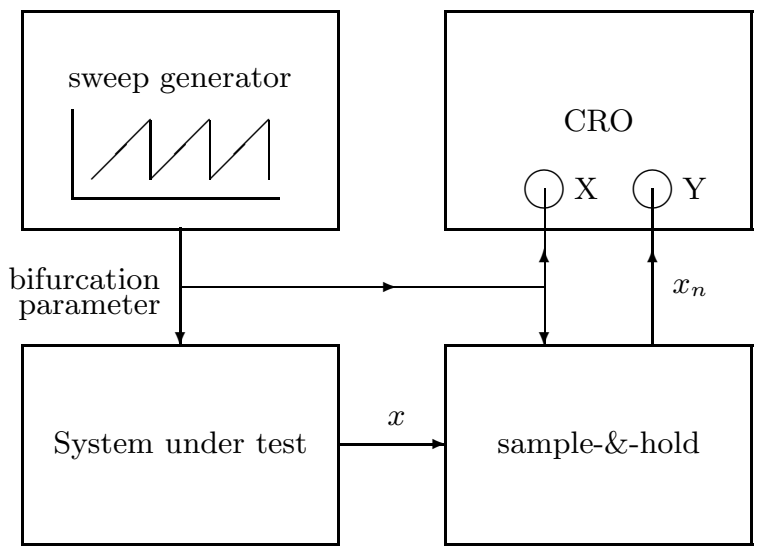

FIGURE 18.9

Block diagram of the system for displaying bifurcation diagrams. $x$ denotes the variable to be sampled from the system under test (SUT). The CRO can be replaced by a computer which acquires the data from the sample-and-hold and the sawtooth generator, and plots/prints the bifurcation diagram.

1. Vary a given parameter of the SUT according to a slowly swept sawtooth voltage which is applied to the $\mathrm{X}$-input of the oscilloscope.

2. Sample a given signal from the SUT and send the sampled data to the Y-input of the oscilloscope.

Moreover, these two functions must be performed in a well coordinated manner. Firstly, the sawtooth must sweep relatively slowly, and the value of the bifurcation parameter is set according to the sawtooth voltage in a stepwise manner. Then, for each value of the bifurcation parameter, the SUT is sampled to give enough data to the Y-input channel. Figure 18.9 shows the functional block diagram of the measurement system.

\subsubsection{Digital implementation and related issues}

We will consider a digital implementation of the required measurement system. The sawtooth voltage can be generated by a $\mathrm{D} / \mathrm{A}$ converter which reads the output from one or more digital counters. The horizontal resolution of the bifurcation diagram is determined by the number of bits of the $\mathrm{D} / \mathrm{A}$ converter. A 12-bit D/A converter, for instance, will offer 4096 steps, and hence will give 4096 points along the horizontal axis of the bifurcation diagram to be displayed on the scope. Figure 18.10 shows the block diagram of a possible implementation of the sawtooth generator. The next question is how fast we 


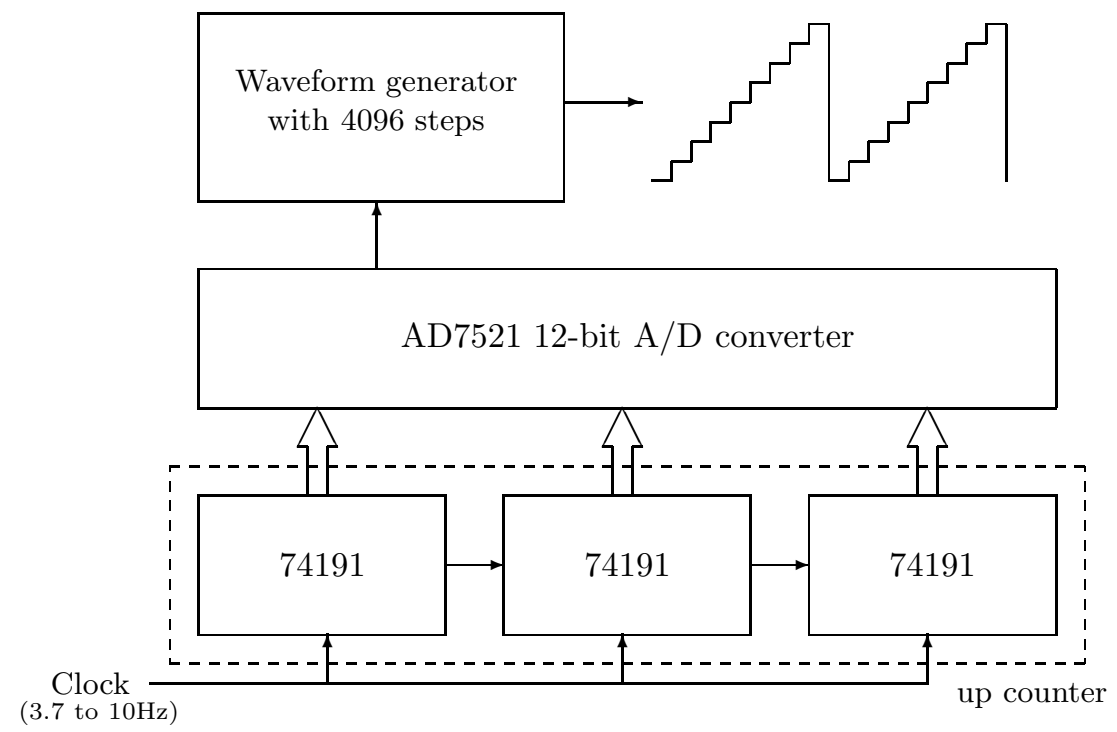

FIGURE 18.10

Block diagram of sawtooth generator. Output serves as voltage analog of bifurcation parameter to be sent to $X$-input channel of oscilloscope and the system under test (SUT).

should drive the counter, i.e., how fast should the sawtooth sweep.

The value of the sawtooth voltage controls the value of the bifurcation parameter used in the SUT. At each step of the sawtooth voltage, we have to ensure that enough time is given to sample enough number of data points from the SUT which are to be sent to the Y-input of the oscilloscope. If the sampling is done at a frequency $f_{s} \mathrm{~Hz}$, and $N$ data points are to be displayed for each value of the bifurcation parameter, then the sawtooth must sweep as slowly as $N / f_{s}$ second per step. Thus, if a 12-bit D/A converter is used, the sweep rate of the sawtooth should be lower than $f_{s} /(4096 N) \mathrm{Hz}$.

Finally, the vertical resolution is controlled by the number of sampled data displayed during each step of the slowly swept sawtooth. Usually 500 samples are adequate. This value, denoted by $N$ above, will affect the sweep rate of the sawtooth.

\subsubsection{Other methods, problems and practical issues}

If the bifurcation parameter is a signal variable (e.g., the reference current), the sawtooth sweep method can be used. But if one intends to study the bifurcations in response to the variation of a power variable (e.g., the input 
voltage) or a physical parameter (e.g., the load resistance), other methods have to be used. There is a simpler way to display a bifurcation diagram on the oscilloscope. The idea is to use the Z-axis modulation to implicitly sample the required variable. This will eliminate the sample-and-hold circuit described above. If the oscillator blanking pulse of the pulse-width-modulator in the converter is available, we may simply use it to drive the Z-input of the scope and hence eliminate the need for constructing a separate driving circuit as mentioned in the previous sub-section. It is worth noting that the use of Z-axis modulation for obtaining bifurcation diagrams is simpler, but is less flexible compared to the use of an extra sample-and-hold circuit which allows the use of a computer for plotting, storing and further manipulating the data obtained from the SUT.

Furthermore, we may generate the sweeping voltage manually with a voltage supply. If we can do it steadily and slowly, we can still get a reasonably good bifurcation diagram. It should be understood that the capturing of the diagram can be done by a DSO, or by a camera using a long exposure time if an analog scope is used.

Finally, there is an important criterion for displaying a bifurcation diagram on the oscilloscope. The bifurcation parameter has to be a voltage or represented by a voltage. In the case where the bifurcation parameter is a current or value of a component (e.g., a resistance), we need to devise a way to make a voltage analog of the bifurcation parameter. This would vary from case to case. For instance, if the load resistance is the bifurcation parameter, we need to produce a voltage analog of the resistance value, sweep it through a suitable range, and feed it to the $\mathrm{X}$-input of the oscilloscope. A handy way to do this is to use a two-limb rheostat with a common jockey. A portion of one limb is connected as the load, and the other limb connected to a separate voltage source. The voltage across the same portion of the second limb is fed to the $\mathrm{X}$-input channel of the oscilloscope. Thus, the variation (i.e., manual sweep) of the load resistance is proportional to the voltage fed to the X-input.

\subsubsection{Example: A simple dc/dc converter}

As an example, we consider a current-mode controlled boost dc/dc converter. The bifurcation parameter is the reference current $I_{\text {ref }}$ which sets the peak value of the inductor current. Figure 18.11 shows the schematic of the converter under study. Our aim is to display the bifurcation diagram, with $I_{\text {ref }}$ as the bifurcation parameter (horizontal axis) and the inductor current as the sampled data (vertical axis).

The operation of the circuit can be described briefly as follows. A $5 \mathrm{kHz}$ clock periodically turns on the power switch. While the switch is on, the inductor current climbs up linearly until its value is equal to $I_{\text {ref }}$ which is the 


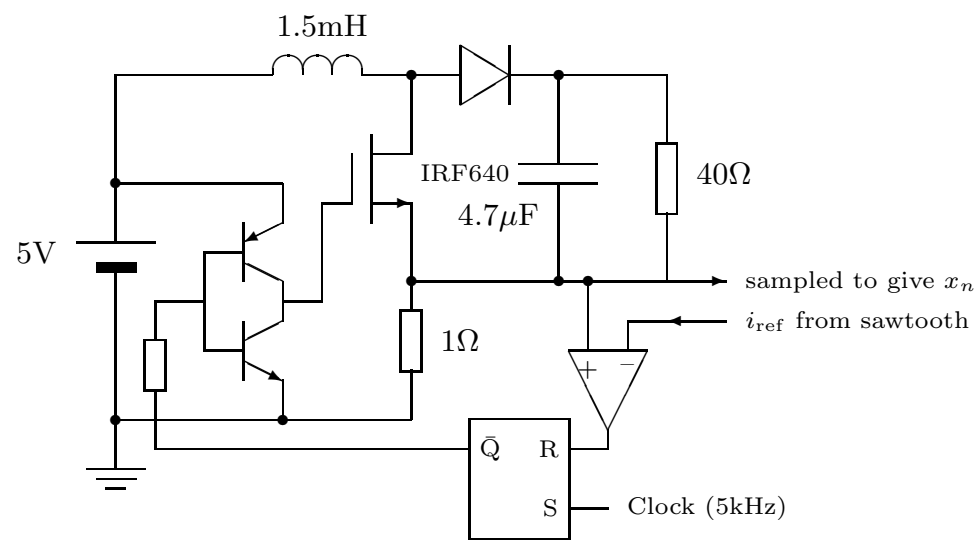

FIGURE 18.11

Schematic of experimental current-mode controlled boost converter, $I_{\text {ref }}$ being the bifurcation parameter supplied by the sawtooth generator. The RS flip-flop block consists of a pair of NOR gates.

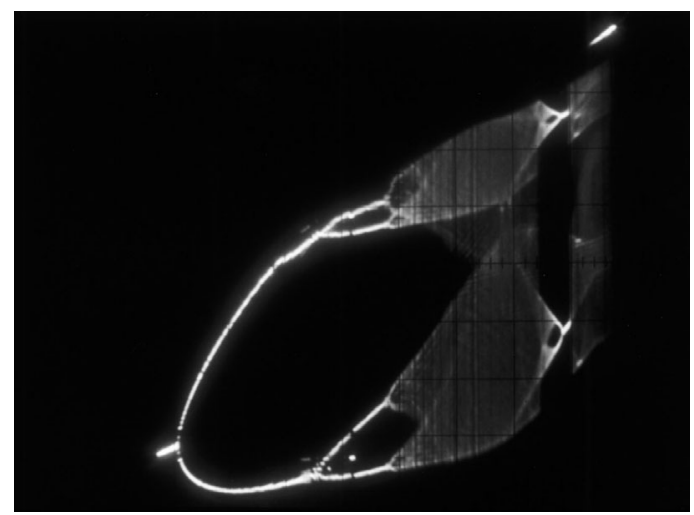

FIGURE 18.12

Bifurcation diagram from oscilloscope for the current-mode controlled boost converter, inductor current being the variable (vertical axis) and peak inductor current $I_{\text {ref }}$ being the bifurcation parameter (horizontal axis). 
bifurcation parameter. When the inductor current reaches (just exceeds) $I_{\text {ref }}$, the comparator goes high, resetting the RS flip-flop. This turns off the power switch. Once the switch is turned off, the inductor current ramps down until the next clock pulse sets the RS flip-flop again and turns the switch back on. The cycle repeats periodically at $5 \mathrm{kHz}$.

The sampling is to be done at the switching frequency of the boost converter, i.e., $5 \mathrm{kHz}$. The variable to be sampled is the inductor current which is picked up by the $1 \Omega$ sensing resistor. The slowly swept sawtooth effectively defines $I_{\text {ref }}$, and is also sent to the X-input of the oscilloscope. 500 samples of inductor current are displayed at each step of the bifurcation parameter.

Figure 18.12 shows a photograph of the oscilloscope display as the trace sweeps horizontally from left to right, corresponding to $I_{\text {ref }}$ swept from 0 to about $1 \mathrm{~A}$. A 12-bit A/D converter is used for the sawtooth generator, i.e., a maximum of 4096 horizontal steps can be recorded. At each step 500 samples are displayed.

Note on Sampling - In the above example, a useful technique on sampling signals from a circuit that involves switching can be noted. Specifically, there are possible ringings (fast oscillatory pulses) sandwiched between smooth segments due to the presence of parasitic inductance and capacitance. When sampling such signals (e.g., inductor current in the above example), care should be taken to avoid sampling at the ringings. We can either apply suitable filtering or deliberately delay the sampling instant. In our example above, sampling is synchronized with the turn-on instants of the power switch, but with a small delay to avoid the ringing pulses.

\subsection{Conclusions}

Many nonlinear phenomena in practical systems are often first observed during experimental measurements and routine simulations, and detailed analysis is done subsequently to provide explanations to the observed phenomena. This mode of investigation is particularly relevant to practical electronic systems. Moreover, if new findings are found analytically, experiments are indispensable for verifying the analytical results. In this chapter, we review some useful laboratory techniques for investigating nonlinear dynamics in electronic circuits. In particular the techniques for displaying phase portraits, Poincaré sections and bifurcation diagrams are discussed in detail. 


\section{References}

[1] N. B. Tufillaro, T. Abbott and J. Reilly, An Experimental Approach to Nonlinear Dynamics and Chaos, Addison-Wesley, New York, 1992.

[2] M. Hasler, "Electrical circuits with chaotic behaviour," Proceedings of IEEE, Vol. 75, No. 8, pp. 1009-1021, August 1987.

[3] S. Prentiss, The Complete Book of Oscilloscopes, McGraw Hill, New York, 1992.

[4] C. W. Wu and N. F. Rulko, "Studying chaos via 1-d maps: a tutorial" IEEE Trans. Circ. Syst. I, Vol. 40, No. 10, pp. 707-721, October 1993.

[5] M. J. Ogorzalek, Chaos and Complexity in Nonlinear Electronic Circuits, World Scientific Series on Nonlinear Science, Vol. 22, 1997.

[6] G. Q. Zhong and F. Ayrom, "Experimental confirmation of chaos from chua's circuits", Int. J. Circuit Theory Appl., Vol. 13, No. 11, pp. 93-98, November 1985

[7] L. O. Chua, C. W. Wu, A. Huang and G.Q. Zhong, "A universal circuit for studying and generating chaos-Part I: routes to chaos", IEEE Trans. Circ. Syst. I, Vol. 40, No. 10, pp. 732-744, October 1993.

[8] L. O. Chua, C. W. Wu, A. Huang and G. Q. Zhong, "A universal circuit for studying and generating chaos-Part II: strange attactors", IEEE Trans. Circ. Syst. I, Vol. 40, No. 10, pp. 745-761, October 1993.

[9] C. K. Tse, S. C. Fung and M. W. Kwan, "Experimental confirmation of chaos in a current-programmed Ćuk converter," IEEE Trans. Circ. Syst. I, Vol. 43, No. 7, pp. 605-607, July 1996.

[10] R. L. Devaney, Introduction to chaotic dynamical systems, Addison-Wesley, New York, 1989

[11] T. S. Parker and L. O. Chua, Practical Numerical Algorithms for Chaotic Systems, Springer-Verlag, New York, 1989.

[12] J. H. B. Deane and D. C. Hamill, "Instability, Subharmonics, and Chaos in Power Electronic Circuits," IEEE Trans. Power Electron., Vol. 5, No. 3, pp. 260-268, 1990.

[13] C. K. Tse, Y. M. Lai and H. H. C. Iu, "Hopf bifurcation and chaos in a freerunning current-controlled Ćuk switching converter," IEEE Trans. Circ. Syst. I, Vol. 47, No. 4, pp. 448-457, April 2000. 